\title{
REACIÓN DE AYUDA A TRAVÉS DE LA HISTORIA: UNA APORTACIÓN DESDE LA PERSPECTIVA ICONOGRÁHCA
}

Mercedes Núñez del Castillo* José Siles G onzález** José Luis Jurado Moyano***

*Profesora Asociada Departamento de Enfermería. Universidad de Alicante, Enfermera Asistencial, U rgencias H ospital U niversitario San Juan de Alicante. **Catedrático de Escuela Departamento de Enfermería. U niversidad de Alicante. ***Profesor Asociado Departamento de Enfermería. Universidad de Alicante. Enfermero Supervisor de D ocencia H ospital U niversitario de San Juan de Alicante.

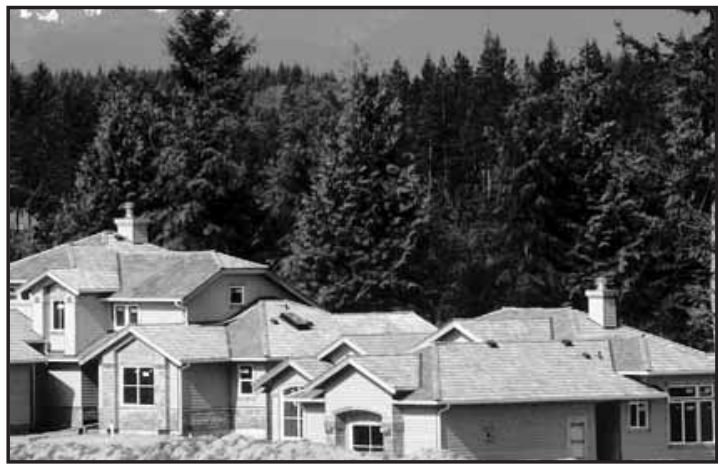

HELP RELATIONSHIP THROUGH HISTORY: A CONTRIBUTION FROM AN ICONOGRAPHIC PERSPECTIVE

\section{SUMMARY}

$\mathrm{H}$ enderson's need to communicate with other people to express emotions, needs, fears or opinions, constitutes the esential basis of help relationship (Rogers, 1986); this help relationship although it might be adapted in a paramount way to nursing care, could not always be totally transferred to those nursing cares. In order to identify help relationship along history, many sources have been used, being iconography one of them.

The iconographic material to be used to verify or invalidate hypothesis is as much of a civil character as of a religious one, obtained by data collected from the National Prado Museum, Madrid Archeological National Museum, and the Cave Paintings around the Mediterranean area, in the Spanish Peninsula. Variables have been distributed in categories according to the characteristics of the so called help relationship. For the purpose of this test, the iconographic interpretation proposed by Panofsky (1995) as well as Siles's diagrams (1999) have been used.

The most ancient iconographies where help relashionship appears are from XI century

and the most recent ones are from XIX century. 6 are from a religious origin and 6 from civil origin.

As a final outcome there could be said, that in relationship verbal or no-verbal communication exists, and that the care taker is always informal.

Keywords: Help relashionship, care, nursing history, iconography.

A RELAÇÃO DE AJUdA ATRAVÉS DA HISTÓRIA: UMA CONTRIBUIÇÃO A PARTIR DA ÓTICA ICONOGRÁFICA

\section{RESUMO}

necessidade de Henderson de se comuni-
car com os outros para expressar emoções,
necessidades, temores ou opiniões constituem as bases essenciais da relação de ajuda (Rogers, 1986). A mencionada teoria, apesar de pode se adaptar de maneira privilegiada aos cuidados de enfermagem, nem sempre pode ser transferida integralmente à assistência. As fontes iconográficas têm sido utilizadas, ao largo da trajetória histórica, para identificar a relação de ajuda.

O material iconográfico que utilizado a fim de verificar ou rechaçar as hipóteses é de procedência tanto civil quanto religiosa, o qual foi obtido por meio de busca iconográfica no Museu Nacional do 
Prado, no Museu Arqueológico Nacional de Madri e Pinturas pré históricas do Arco Mediterrâneo da Península Ibérica. As variáveis foram distribuídas por categorias e correspondem às características da relação de ajuda. Para a análise, foi utlizada a interpretação iconográfica proposta por Panofsky (1995) e os esquemas propostos por Siles (1999).

As iconografias mais antigas, nas quais se identifica a relação de ajuda, são do século XI e as mais recentes são do século XIX, sendo 6 delas de natureza religiosa e 3 de natureza civil.

Como conclusão, pode-se dizer que há tanto comunicação verbal quanto não verbal e que o cuidador é sempre informal.

Palavras-chave: Relação de ajuda, Cuidados, história da enfermagem, iconografia.

\section{RESUMEN}

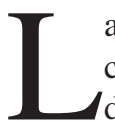

a necesidad de Henderson de comunicarse con otros para expresar emociones, necesidades, temores u opiniones, constituyen las bases esenciales de la relación de ayuda (Rogers, 1986); ésta, a pesar de que puede adaptarse de forma privilegiada a los cuidados de enfermería, no siempre puede transferirse íntegramente a estos. Para identificar la relación de ayuda a lo largo de la historia se han utilizado entre otras fuentes, las iconográfícas.

El material iconográfico que se va a utilizar para verificar o refutar las hipótesis es tanto civil como religioso, obtenido mediante búsqueda iconográfica, en el Museo Nacional del Prado, Museo Arqueológico Nacional de Madrid y Pinturas rupestres del Arco Mediterráneo de la Península Ibérica, Las variables, están distribuidas por categorías y corresponden a las características de la relación de ayuda. Para el análisis, se ha utilizado la interpretación iconográfica propuesta por Panofsky (1995) y los esquemas propuestos por Siles (1999).

Las iconografías más antiguas donde aparece la relación de ayuda son del siglo XI y la más reciente del XIX; 6 son de naturaleza religiosa y 3 de naturaleza civil.

Como conclusión, se puede decir que hay tanto comunicación verbal como no verbal, y que el cuidador es siempre informal.
Palabras clave: Relación de ayuda, cuidados, historia de la enfermería, iconografía.

\section{INTRODUCCIÓN}

La necesidad de Henderson de comunicarse con otros para expresar emociones, necesidades, temores u opiniones, constituyen las bases esenciales de la relación de ayuda (Rogers,1986) entre las definiciones que aporta sobre el tema, afirma: "..es aquella en la que uno de los participantes intenta hacer surgir, de una o ambas partes, una mejor apreciación y expresión de los recursos latentes del individuo, y un uso más funcional de éstos.." (Bermejo, 1996); la relación de ayuda a pesar de que puede adaptarse de forma privilegiada a los cuidados de enfermería, no siempre puede transferirse íntegramente a estos, sobre todo en el caso de la entrevista estructurada. Siguiendo a Madrid: La relación de ayuda es una experiencia humana privilegiada que ofrece el marco adecuado para facilitar el desarrollo de las capacidades bloqueadas. (Bermejo, 1996).

Y Riopelle, la define como un intercambio "siendo a la vez verbal y no verbal, que permite crear un clima de confianza, de respeto y de amor que el cliente precisa para satisfacer sus necesidades fundamentales, para alcanzar un contacto mejor con su propia realidad, sus emociones, sus conflictos, sus valores, sus límites y sus aspiraciones"(Phaneuf, 1982). Cibanal, (2003), clarifica el concepto, señalando, que consiste en comunicarnos en dos lenguajes: el de hablar y el de escuchar, en darle el protagonismo a las personas y el papel justo a las técnicas y los medios, utilizándolos como habilidades y recursos que están al servicio de la persona; siendo la persona el objetivo de los cuidados y el servicio a las mismas debe ser el núcleo de las actividades de enfermería como afirma la Dra. Henderson.

El clasificar los tipos de relación de ayuda, puede resultar un poco tópico, pero para el contexto de la investigación va a resultar útil, y por tanto definiremos para los cuidados enfermeros dos tipos de relación de ayuda, la formal y la informal. La relación de ayuda de tipo informal es la que se establece simplemente observando las necesidades del momento (mirada y, o aspecto triste, lamentos), siendo espontánea y amoldándose a la situación, 
estableciendo una relación de confianza, observando los comportamientos no verbales como son las escucha y la empatía. En cuanto a la relación de ayuda de tipo formal, ya no se trata de responder de forma inmediata a las necesidades del paciente, sino de aplicar técnicas que le ayudarán a superar la dificultad que tiene, observándose varias fases.

Hay una serie de actitudes en el profesional de enfermería que son fundamentales para la relación de ayuda son las actitudes facilitadoras, como es la escucha activa y unas actitudes elaboradoras como son el proceso de acogida, que implica un proceso de aceptación, el respeto cálido y la comprensión empática que se refiere a la característica que aparece de forma espontánea entre las personas.

La escucha activa pone en funcionamiento una serie de comportamientos cuyo objetivo es que el paciente sepa que se está ahí, bien con la mirada, la postura o los gestos, ayudando a la enfermera a comprender lo que siente la persona ayudada y confirmando que entiende el mensaje, utilizando el mecanismo de la reformulación o efecto espejo.

El proceso de acogida, implica un proceso de aceptación, haciendo sentirse al paciente cualquiera que sea su estado, que la enfermera está ahí tendiendo la mano de la ayuda; la relación de ayuda se apoya en el respeto y confianza, así en el respeto cálido las actitudes de comunicación del profesional de enfermería son el contacto visual, atención a lo que dice, o postura adecuada, en la comprensión empática se refiere a la característica que aparece de forma espontánea entre las personas, situándose

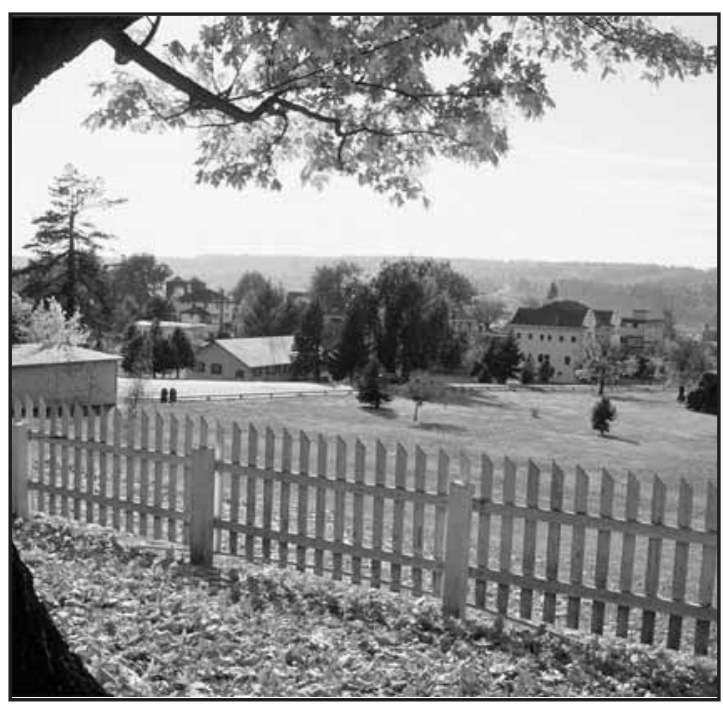

en un nivel de comprensión que sobrepasa al mismo problema, se centra en la subjetividad del otro, en su mundo emotivo, refiriéndose por ejemplo a una mirada interesada o a una voz cálida.

La relación de ayuda pasa por diferentes fases, en las que las habilidades del profesional se manifiestan de forma diferente dependiendo del tipo de relación de ayuda, Peplau, propone cuatro fases o etapas (Cibanal, 2003). En este trabajo se han considerado solo tres; en la relación de tipo informal en la primera fase el profesional observa el comportamiento verbal o no verbal del paciente en donde se ve la necesidad de ayuda, (aspecto triste, mirada, lamentos); en la segunda fase es el momento del establecimiento de la comprensión empática con el paciente y en la tercera fase se realiza la conclusión, y no debe terminarse sin delicadeza y sin tener seguro que las intervenciones han sido positivas.

A partir de la lectura, la observación o mediante experiencias prácticas vemos que existen iconografías en las que aparece la relación de ayuda. Para ver la relación de ayuda a lo largo de la historia entre otras fuentes, se han utilizado iconografías, pintura, escultura o cualquier otra fuente histórica que se nos presente, como dicen algunos autores monumentos silenciosos del pasado.

Por tanto las fuentes históricas que se van a analizar son fuentes directas. Pero además de ver, el observador necesita saber si están ahí para influir sobre los conocimientos, necesidades u opiniones de algunas personas (fuentes con o sin destinatario), ya que el hombre es un animal que recuerda, y como recuerda tiene experiencia y como la tiene, proyecta. Un hombre que careciera de toda vinculación con el pasado sería incapaz de proyectarse hacia delante, le faltaría la conciencia misma de que existe un futuro (Chueca Gotilla, 1987).

Estas fuentes iconográficas reflejan y transmiten a la sociedad y la cultura de ese momento, aportando datos importantes sobre el pasado de la actividad cuidadora.

A lo largo de los siglos, con el difícil acceso del pueblo a la cultura y el saber, es probable que estas fuentes fueran la forma silenciosa y a la vez viva de hacer llegar la información necesaria para mantener la supervivencia. Por tanto resulta interesante conocer la forma de expresión artística que se 
utiliza desde entonces para saber quienes somos y que cuidados hemos dado.

Santo Tomás, opina que “..investigar en historia, implica saber utilizar los instrumentos que el tiempo ha ido poniendo a nuestra disposición..","..aprender a interpretar los testimonios que otros hombres y mujeres de épocas pasadas nos han dejado como legado..", sigue diciendo esta autora más adelante “..existen además otro tipo de fuentes que son manifestaciones artísticas.....estas fuentes llamadas iconográficas..”. “..Es por tanto la iconografía una fuente importante que podemos y debemos utilizar las enfermeras que deseamos conocer nuestro pasado.." (Santo Tomas, 1997).

\section{OBJETIVO}

Describir y analizar la relación de ayuda a lo largo de la historia desde la perspectiva de V. Henderson mediante el análisis iconográfico.

\section{HIPÓTESIS}

Y como punto de partida pensamos que:

- La iconografía constituye una fuente para analizar la relación de ayuda desde la perspectiva de Virginia Henderson.

- La relación de ayuda, esta presente a lo largo de la historia en las fuentes iconográficas.

\section{ESTADO DE LA CUESTIÓN}

La investigación relacionada con la historia de la salud y de la enfermedad ha sido tratada tradicionalmente por historiadores de la medicina que han utilizado tanto fuentes escritas como iconográficas, lo que ha movido a investigadores de otras disciplinas a trabajar otros aspectos de la misma historia, bien sanitarios, sociales, culturales o de cuidados enfermeros. Santo Tomás (2002), afirma que aspectos de salud-enfermedad influyen en la vida de un grupo social, en un momento determinado, tanto como influyen otras causas de aspecto físico, social o religioso, constituyendo todos estos aspectos un enriquecimiento para los conocimientos de la historia en general y de la historia de la enfermería en particular.

Intentamos relacionar la enfermería, la relación de ayuda o comunicación terapéutica y la iconografía como instrumento para la investigación, y para ello vamos a utilizar un método que consideramos de plena actualidad que es propuesto por

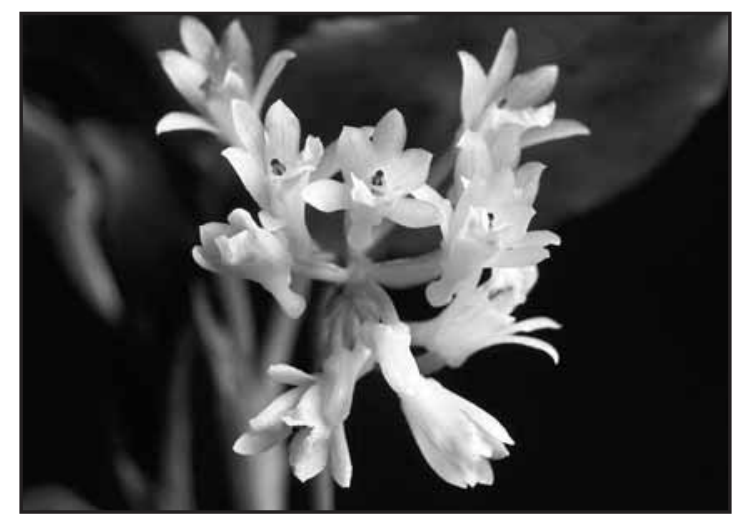

Panofsky (1995), y que Santo Tomás (1996, 1997), además de argumentar la importancia de la iconografía para el estudio de la historia de la enfermería, propone una metodología para el estudio de las iconografías, y, que es el propuesto por Panofsky, (1995). Siles, (1999) modifica el método desarrollado por Panofsky y lo adapta a la cultura enfermera; Núñez, (2004,2006) y Morillo, (2005) utilizan para el análisis iconográfico de los artículos consultados el propuesto por Panofsky y modificado por Siles. Correia Hirata, (2003), en "Iconografía e enfermagem - por que e como?", afirma que utiliza la iconografía como una fuente para el estudio de la enfermería, justificando como ha de estudiarse una obra de arte para que de pistas sobre la actividad cuidadora; cita ejemplos de los momentos del análisis iconográfico y el método usado para observar el significado profundo de la obra de arte

Algunos autores como Lagraña (2001) y Lussiez (2005), solo hacen indicación de la observación de las obras de arte para utilizarlas como un elemento más para el análisis y estudio del tema.

González (2003), considera el método de iconodiagnóstico como un método complementario de la enseñanza. Correia Hirata (2003), argumenta el uso de la iconografía como una fuente para el estudio de la enfermería, justificando como ha de estudiarse una obra de arte para que nos de pistas sobre nuestra actividad cuidadora; Riley (2004), utiliza la fotografía en la práctica de enfermería clínica, clasificando en categorías las diferentes aplicaciones para las cuales la fotografía había sido aplicada (documentación y vigilancia, intervención terapéutica, aprendizaje, docencia y evaluación), concluyendo en su investigación que puede 
ser utilizada como método para docencia, aprendizaje o como método de observación y Wang (2004), también utiliza la fotografía como herramienta pedagógica para mejorar la profesionalidad de los estudiantes de medicina.

Pardue (2005), utiliza las galerías de arte como seminario para el aprendizaje de alumnos de enfermería, estimulando el desarrollo de habilidades mediante la observación de las obras de arte y su análisis iconográfico, y Kirklin (2007), a través de un estudio comparativo realizado con médicos y enfermeras, y cuyo objetivo ha sido averiguar si perfeccionan sus habilidades a través de la observación de fotos, concluyendo que la rama de intervención las mejoró y; también Arnold (2006), a través de la biografía de Van Gogh, incluida la patología del artista, analizando y estudiando sus obras, ha creado una Web con pinturas e ilustraciones, cuyo objetivo principal es que sirva para la educación de los alumnos de medicina. Nelson, et al (2006), consideran las fotografías de procesos patológicos (quemaduras), útiles para el aprendizaje de los miembros de un equipo multidisciplinar, al poder observar la valoración y evolución de ciertas heridas y complicaciones.

\section{METODOLOGÍA}

El área de estudio se centra en España. El material iconográfico que se va a utilizar para verificar o refutar las hipótesis es tanto civil como religioso, obtenido mediante búsqueda iconográfica, en el Museo Nacional del Prado, Museo Arqueológico Nacional de Madrid y Pinturas rupestres del Arco Mediterráneo de la Península Ibérica, analizándolo desde principio de la historia hasta nuestros días, y organizados por categorías como son: pintura y escultura en todas su formas.

Las variables, también están distribuidas por categorías y corresponden a las características de la relación de ayuda como forma de comunicación terapéutica, actitudes, tanto facilitadoras como elaboradoras, fases de la relación y técnicas.

Los datos cualitativos se han recogido a través de la observación de las obras de arte seleccionadas. Para analizar las iconografías, se llevan a cabo dos tipos de análisis; el iconográfico, propiamente dicho, y el antropológico.

Para realizar el, análisis, se ha utilizado la interpretación iconográfica propuesta por Panofsky
(1995) y los esquemas propuestos por Siles (1999); para el análisis cualitativo de datos se ha utilizado el programa ATLAS/ti, versión 4.1 para Windows.

\section{RESULTADOS}

Se han analizado 87 obras de arte, desde el neolítico hasta el primer tercio del siglo XX.

El número de iconografías donde aparece la relación de ayuda son 9 , siendo las más antiguas del siglo XI y la más reciente del XIX; 6 son de naturaleza religiosa y 3 de naturaleza civil, 6 de ellas pertenecen a la escuela española y 3 a la flamenca.

En la arqueta de San Felices, formada con placas de marfil, del siglo XI, en una de estas piezas y distribuida en dos partes, en la inferior, se representa la curación del hijo de la viuda y en la superior la madre agradeciendo al santo su intervención, interpretándose como un momento de escucha activa; el grupo iconográfico del santo con la madre y el hijo en el momento del milagro también se puede interpretar como respeto cálido, por las actitudes de ambos.

El arca de San Millán de la Cogolla, relieve del siglo XI, mozárabe de naturaleza religiosa como la anterior, formado también por placas de marfil, se observa al ciego que se aproxima y toca el relicario ayudado por otro hombre que va detrás de él, interpretándose éste como un momento de acogida. En otra placa de marfil de la misma arca se observa un momento de escucha activa entre el santo y la paralítica recién curada con el milagro.

En "la tabla de los pecados capitales" de El Bosco, perteneciente a la escuela flamenca del siglo XV y considerada como pintura religiosa, nos interesa analizar dos detalles, en un lateral aparecen unas mujeres recogidas, sentadas alrededor de una mesa, en señal de recogimiento por el duelo al que acompañan, interpretando el momento como una primera fase de la relación de ayuda informal. El segundo detalle es la soberbia representada por una mujer que se mira al espejo, interpretándose como una técnica de reformulación.

En "El descendimiento de la Cruz" de Van der Weyden, pintura flamenca del siglo XV, también de naturaleza religiosa, se adivina la actitud de acogida de las Santas mujeres y San Juan que sostienen a la Virgen desfallecida por el dolor de la pérdida de su Hijo. 
"La adoración del sepulcro de San Pedro mártir", de Berruguete, del siglo XV, observamos dos actitudes, la empatía y el respeto cálido por la confianza y el respeto que hay entre el ciego y el lazarillo.

En el otro "Descendimiento" español del siglo XVI de Machuca se observa también una actitud de respeto cálido que al igual que la anterior la Virgen con gran dolor en la cara y medio desmayada es sostenida por María Magdalena y San Juan para evitar que se caiga.

En el lienzo de Teniers, "La intervención", del siglo XVII, flamenco, se interpreta como una actitud de acogimiento y la escucha activa entre profesional y paciente. El cirujano parece ayudar al paciente a captar y hacer frente a sus problemas, es como que con su mirada ayuda a disminuir la ansiedad, da la impresión de que están solos.

En "La Recuperación de la Bahía de Dos Santos", de Maino, del siglo XVII, de escuela española y naturaleza civil como la anterior, se interpreta como una acogida al moribundo por parte de los lugareños.

En la pintura "Doña Juana La Loca," de Pradilla, del siglo XIX, y de naturaleza civil, se interpreta una actitud de empatía de los componentes del séquito hacia la dama.

\section{DISCUSIÓN}

Atendiendo a los objetivos del estudio, se analizan los resultados más llamativos y las diferencias entre los datos referentes a la relación de ayuda en las iconografías analizadas. Consideramos que el tipo de relación de ayuda que se observa es de tipo informal pues surge de forma espontánea, estableciéndose según la necesidad del momento y los objetivos se adaptan a la situación y tiempo, siendo reflejo puntual de un momento que plasma el artista. Se puede decir que hay tanto comunicación verbal como no verbal, y que el cuidador es siempre informal.

\section{ACTITUDES FACILITADORAS}

Respecto a la escucha activa que aparece en tres iconografías vemos que hay actitud de compartir en ambos casos, demostrando al ayudado que está ahí, observándose miradas, gestos y posturas, pero por el contrario no se puede ver el mecanismo de la reformulación. En los relieves del arca de San Millán y en la arqueta de San Felices, el agradecimiento como efecto de la ayuda recibida, de haber escuchado las súplicas para la curación, hay un intercambio, pues la paralítica y el hijo de la viuda se han curado y el santo se ha engrandecido. En La operación de Teniers, hay un estado de receptividad en el paciente, donde el profesional le ayuda a captar y hacer frente a sus problemas.

\section{ACTITUDES ELABORADORAS}

En el respeto cálido hay tres referencias, se observan únicamente las actitudes propias de la comunicación que demuestran al cliente o ayudado toda la consideración que el cuidador le concede como es el contacto visual o la atención a lo que dice, postura, actitud de respeto y confianza en adoración al sepulcro de San Pedro, mártir, Arca de San Felices, Descendimiento de Machuca.

De la empatía, bien como actitud o como fase hay dos referencias, que resultan difícil de interpretar y nos tenemos que centrar en las evidencias del ayudado, es decir lo que expresa explícitamente e intentar buscar sus sentimientos, y solo vemos las miradas en el comportamiento no verbal y en el contacto de las personas tanto del ayudado como del cuidador, en "Doña Juana la Loca”, el nivel de comprensión sobrepasa el problema, entendiendo en el ayudado su manera de vivir y pensar la dificultad; interpretándose como una actitud de empatía de los componentes del séquito hacia la dama; en la "Adoración al sepulcro de San Pedro", hay miradas de cariño y manera de acompañar y sujetar el cayado y como se apoya el peregrino en el lazarillo (Acompañamiento), no hay comunicación verbal, pero si contacto que resulta igual de útil. Según afirma (Bermejo, 1998) podemos inferir, como en algunas iconografías el ayudante o cuidador penetra en la experiencia que el otro está viviendo, llegando incluso a identificarse con él.

En cuanto a la acogida como una primera fase de la relación de ayuda informal hay seis referencias, principio de aceptación en el arca de San Millán donde el ayudante le tiende la mano segura, es un hombre que camina detrás del ciego ayudándole a llegar hasta el relicario, tanto en el arca de San Millán o en la adoración del sepulcro de San Pedro mártir, el hecho de que el ciego toque el relicario puede interpretarse como un recurso, la fe; en 


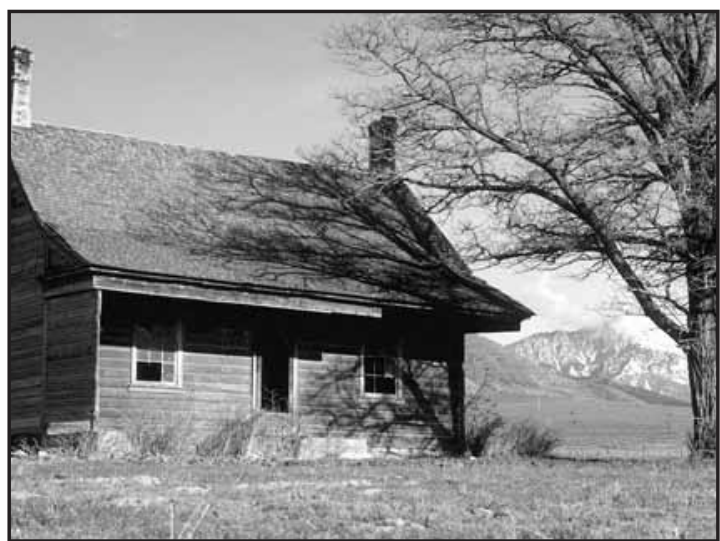

algunos casos, éste y otros recursos quedan secuestrados y cuando se descubren sirven de ayuda, así mismo el hecho de apoyarse en alguien también le sirve como guía para que utilice esos recursos propios. En la soberbia del Bosco, con la visión de si misma en el espejo, éste puede significar el propio ayudante, en lo positivo y en lo negativo.

En los Descendimientos de la Cruz de Van der Weyden y Machuca miradas de tristeza y de gran dolor en la Virgen que es ayudada por Maria Magdalena y San Juan; solo nos podemos centrar en el comportamiento no verbal de las dos partes, quedando reflejado y observándose la necesidad de ayuda en la mirada o el aspecto triste, .Acompañamiento y respeto en el momento en que se aproxima la muerte en la Tabla de los pecados capitales. En La operación de Teniers el profesional, ayuda al paciente a captar y hacer frente a sus problemas, con la mirada le ayuda a disminuir la angustia y preocupación, da la impresión de que están solos. En la Recuperación de la Bahía de dos Santos, hay una acogida al moribundo por parte de los lugareños.

Respecto a las fases de la relación de ayuda informal, en la primera fase, hay un comportamiento no verbal de la persona que revela la necesidad de ayuda, con la mirada de esperanza del ciego y la del lazarillo que le atiende en la Adoración al sepulcro de San Pedro mártir, también se podría interpreta una primera fase del acercamiento en el acompañamiento a un duelo, en la Tabla de los pecados capitales, la acogida también enumerada anteriormente.

En la segunda fase que se entiende la empatía como una fase, ya se ha enumerado.
En la tercera fase o de conclusión hay dos referencias, en el Arca de San Felices y en la de San Millán, aparecen los ayudados agradeciendo la ayuda recibida, sujetando entre sus manos las del santo.

En cuanto a técnicas, la única que se ha observado es la de reformulación, bien como técnica o como actitud, en la tabla de los pecados capitales, donde se observa la percepción que tiene la mujer de si misma, que es captada, transmitida y observada en el espejo donde se mira.

\section{CONCLUSIONES}

La iconografía constituye una fuente para analizar la relación de ayuda desde la perspectiva de Virginia Henderson.

La relación de ayuda, esta presente a lo largo de la historia en las fuentes iconográficas, siendo el siglo XI, la primera iconografía referencia en la muestra estudiada.

Es un trabajo pionero en el marco de la Enfermería al instrumentalizar un Modelo de Enfermería (Henderson), para realizar una interpretación iconográfica de la enfermería. Por lo que hay que profundizar en esta temática vinculando la iconografía y los cuidados de salud.

\section{BIBLIOGRAFÍA}

- ARNOLD, W. (2006): Were Art and Science Converge: The illness of Vincent van Gogh. 12 may. En http://www.kumc.edu/new/publish/article_00773.shtml Consultado 20/5/2007.

- BERMEJO,J.C, (1996). Apuntes de relación de ayuda. Cuadernos del Centro de Humanización de la Salud.

- BIEDERMANN, H. (1993):Diccionario de símbolos. Paidós, Barcelona.

- CALVO, S (2000): El descendimiento de Roger Van der Weyden. (obtenido el 17 marzo, 2005). Centro Virtual Cervantes. Instituto Cervantes (España) 1998-2005 http://cvc.cervantes.es

- CAMÓN, J. (1966): La pintura medieval española. Summa Artis. Historia General del arte. Volumen XXII, primera edición. Espasa Calpe S.A. Madrid. Capítulo libro.

- CIBANAL, L, ARCE, M. C., CARBALlAL, M. C. (et al). (2003): Técnicas de comunicación y relación de ayuda en ciencias de la salud. Editorial Elsevier España.

- CORREIA, M. (2003): iconografía e enfermagem - por que e como?. Online Brazilian Journal of nursing (OBJN-ISSN 1676-4285) v.2,n.2 online. www.uff.br/nepae/objn202hirata.htm obtenido 29 julio 2005.

- DE ARMAS, I (2001): Cara y cruz del dolor. Ed. Laboratorios Menarini. Madrid.

- CHUECA GOTILLA, F. (1987): Prólogo. Gran historia universal volumen I Prehistoria. Cano Herrea, M; Chapa Brunet, 
T; Delibes de Catro,G; Moure Romanillo,JA; Querol,MA; Santonja Gomez,M. Editorial Najera, Madrid.

- FORD, JMT. (2004): Florence Nightingale, OM (1820-1910). Santa Croce, Florence, and St. Margaret of Antioch, East Welow, Hampshire. Journal of Medical Biography. May 12:120.

GONZÁLEZ, F. (2003): L./A. Le main: Tabaquismo. Jano. Vol. LXIV (1479): 79-80.

HENDERSON, V. A. (1994): La naturaleza de la enfermería. Una definición y sus repercusiones en la práctica, la investigación y la educación. Reflexiones 25 años después. Mc Graw-Hill Interamericana de España S.A.

- KIKLIN, D; J, DUNCAN; S, MC.BRIDE; S. HUNT; M,GRIFFIN. (2007): A cluster design controlled trial of artsbased observational skills training in primary care. Medical Education. Vol. 41, nº 4 April: 395-401.

- LAGRAÑA, R; ANTONIO TERRAES; DIOCLES REVIDATTI; DIOCLES (H). (2001): Anatomía de la crucifixión. Las manos Ciencias médicas. Comunicaciones científicas y tecnológicas.

- LUSSIEZ, B (2005): Anatomie de la crucifixion. Chirugie de la main. V. 24, (3-4): 132-147.

- MADRID SORIANO, J: (1986). Relación de ayuda y comunicación en AA.VV Hombre en crisis y relación de ayuda. Asetes. Madrid.

- MONTORO, J. (1992): Los ciegos en la historia. Tomo II. ONCE. Organización Nacional de Ciegos Españoles. Dirección General, sección de cultura. Madrid.

MORILLO, R.M. (2005): La enfermedad de la locura a través de la mirada de Goya .Evidentia. Resumen de comunicaciones. VII reunión sobre Investigación cualitativa en salud. IV jornadas internacionales sobre Cultura de los cuidados. II reunión de enfermeras y enfermeros antropólogos. Investigación cualitativa+cultura de los cuidados conocimiento humanista. Evidentia 2005 sep-dic; 2(6). En http://www.index-f.com/evidentia/n6/157articulo.php [ISSN: 1697-638X], consultado el $15 / 4 / 2007$.

- NELSON, L (ET AL). (2006): Are burns photographs useful?. Burns. Nov; 32 (7): 876-879. Epub sep 25.

- NOGALES, A. (1996): La Edad Media: Los orígenes de la Enfermería. II parte del libro: Historia de la Enfermería en España. (Desde la antigüedad hasta nuestros días), coordinadora del libro Francisca Hernández Martín.

- NUÑEZ, M; SILES, J. (2004): Evolución de los cuidados enfermeros. Análisis iconográfico desde la perspectiva de Virginia Henderson. Cultura de los cuidados. (15): 17-25.

- (2004): Tras las huellas de nuestros orígenes cuidadores. Cultura de los cuidados. (16): 14-19.

- (2004): El dolor en el arte, análisis iconográfico de las necesidades de Henderson. Sociedad Española de Enfermería neurológica.(20): 18-23.

- PANGRAZZI,A (2000): Girasoles junto a sauces. En diálogo con los enfermos. Editorial Sal Terrae Santander.

- PARDUE, K.T. (2005): Blending aesthetics and empirics: teaching health assessment in an art gallery. J. Nurs Ed. Jul; 44 (7): 334-337.

- PANOFSKY,E (1995): El significado en las artes visuales. Alianza Editorial.

- PHANEUF, M (1982) La comunication et la relation d'aide : éléments de compétence de $1^{\prime}$ infirmière, $2^{\text {a }}$ parte, Nursing
Quebec, Vol $2: 3$ marzo, abril.

- RILEY, RG Y E. MANIAS. (2004): The uses of photography in clinical nursing practice and research: a literature review. J. Adv Nurs Nov; 48 (4): 397-405

- ROGERS, C R.(1986): El proceso de convertirse en persona. Paidos, Barcelona.

- SANTO TOMÁS PÉREZ, M. (2002): “Asistencia a los enfermos en Castilla en la Baja Edad Media". Tesis doctoral. Biblioteca virtual Miguel de Cervantes

- SANTO TOMÁS PÉREZ, M. (et al). (1996): Iconografía: un instrumento para la investigación histórica. Index Enferm V(16-17):87-88 .

- (1997):De la muy activa vida de las monjas del Hôtel Dieu de Paris: análisis iconográfico de la miniatura del siglo $\mathrm{XV}$ " Index de Enfermería; 19 : 45-48

- (1997): "Iconografía y enfermería : un instrumento para la investigación histórica" Index de Enfermería; 19 : 13-16.

- SANTO TOMÁS PÉREZ, M, MELCHOR, A., CASTANBEDO, C., FERNANDEZ, M. L., GONZALEZ, I. (1996): Iconografía . Un instrumento para la investigación histórica. Index de Enfermería. (16-17): 87-88.

- (1997): Iconografía y Enfermería. Un instrumento para la investigación histórica. Index de Enfermería. (19), 13.

- SANDRIN-BRUSCO (et al). (1992): Comprender y ayudar al enfermo. Editorial San Pablo.

- SILES GONZÁLEZ, J. 1999: Historia de la Enfermería volumen I y II. Consejo de enfermería de la Comunidad Valenciana.

- SUNDEEN, S. J. (et Al). 1981: L'interazione infermierepaziente. Il processo di nursing. Ambrosiana, Milán.

- TAN S.Y. Y P. HOLLAND. (2006): Florence Nightingale (1820-1910): founder of modern nursing. Singapore Med J. Mar; 47 (3): 185-6.

- Una obra, un artista. Mirar un cuadro: Mesa de los pecados capitales. Hieronymus Van Aeken Bosch "El Bosco".Mayo 2003.

- Descendimiento de la Cruz. Roger Van der Weyden.

- El descendimiento de la Cruz. Pedro Machuca.

- http://museoprado.mcu.es

- VENDRAME, C. (2002): Los enfermos en la Biblia. Editorial San Pablo, Madrid.

- WANG,C.C.; C.A. PIES. (2004): Family, Maternal, and Child Hearth Trough Photovoice. Maternal and Child Hearth Journal, 8 (2).

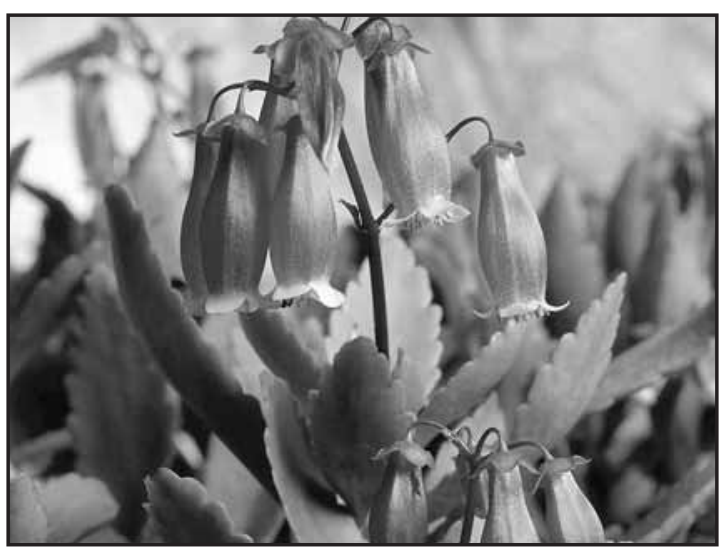

